\title{
Densidad de alojamiento, hematocrito y relación heterófilo/linfocito en pollos parrilleros en las cuatro estaciones del año
}

\author{
Gallard, E.A.'; Menichelli, M.L.'; Dimasso, R.J.; Revidatti, A.F. ${ }^{3}$ \\ ${ }^{1}$ INTA EEA Reconquista, RN11, Km 773, 3560, Reconquista, Santa Fe. \\ ${ }^{2}$ Prof. Honor. Fac. Cs. Veterinarias, UNR. ${ }^{3}$ Cátedra Prod. Aves, Fac. Cs. Vet. UNNE. \\ E-mail: gallard.eliana@inta.gob.ar
}

\begin{abstract}
Resumen
Gallard, E.A.; Menichelli, M.L.; Dimasso, R.J.; Revidatti, A.F. Densidad de alojamiento, hematocrito y relación heterófilo/linfocito en pollos parrilleros en las cuatro estaciones del año. Rev. Vet. 32: 2, 164-168, 2021. Las variaciones del entorno avícola derivadas de la densidad de alojamiento influyen sobre el bienestar animal, cuyo deterioro se refleja en distintos indicadores hemáticos. El objetivo de este trabajo fue evaluar el efecto de la reducción en la densidad de alojamiento sobre el hematocrito y la relación heterófilo/linfocito $(\mathrm{H} / \mathrm{L})$ en pollos parrilleros machos y hembras en las cuatro estaciones del año. Se trabajó con pollos híbridos Cobb500 en lotes mixtos, en galpones oscurecidos de ventilación forzada tipo túnel. Las densidades de alojamiento aplicadas fueron: estándar $\left(14 \mathrm{pollos} / \mathrm{m}^{2}\right)$ y reducida $\left(12 \mathrm{pollos} / \mathrm{m}^{2}\right)$. En el sacrificio a los 42 días de edad se tomaron muestras de sangre anticoagulada de 30 machos y 30 hembras de cada tratamiento, determinándose el hematocrito por el método micro y la relación $\mathrm{H} / \mathrm{L}$ por el recuento de heterófilos y linfocitos mediante frotis. Los datos se evaluaron con la prueba $U$ de Mann-Whitney en forma separada para cada sexo y estación, con la densidad como fuente de variación. El valor del hematocrito mostró diferencias significativas a favor de las hembras alojadas con densidad estándar en el invierno (estándar 32,5 vs reducida $31 \%, p=0,035)$ y una diferencia marginalmente significativa para este grupo experimental en el ensayo de primavera (estándar 31 vs reducida 30\%; p=0,093). La H/L mostró diferencias marginalmente significativas en los machos con valor más alto en la densidad reducida en verano $(\mathrm{p}=0,093)$ y en las hembras en verano $(\mathrm{p}=0,08)$ y en invierno $(\mathrm{p}=0,077)$. Se concluye que la modificación de la densidad de alojamiento en invierno genera condiciones ambientales que derivan en un aumento del hematocrito en los pollos alojados a mayor densidad, respuesta que es más evidente en el caso de las hembras, en tanto que la disminución de la densidad produce aumentos de la $\mathrm{H} / \mathrm{L}$ no compatibles con situaciones de estrés crónico.
\end{abstract}

Palabras clave: pollos parrilleros, bienestar animal, estrés, hematología.

\begin{abstract}
Gallard, E.A.; Menichelli, M.L:; Dimasso, R.J.; Revidatti, A.F. Stocking density, hematocrit and heterophil/ymphocyte ratio in broiler chickens in the four seasons of the year. Rev. Vet. 32: 2, 164-168, 2021. Variations in poultry environment derived from stocking density influence animal welfare, whose deterioration is reflected in different hematic indicators. The objective of this study was to evaluate the effect of the reduction in stocking density on the hematocrit and the heterophile:lymphocy te ratio $(\mathrm{H} / \mathrm{L})$ in male and female broiler chickens in the four seasons of the year. Cobb500 hybrid chickens were reared in mixed batches, in dark houses tunnel forced ventilation. The stocking densities were standard $\left(14\right.$ chickens $\left./ \mathrm{m}^{2}\right)$ and reduced (12 chickens $/ \mathrm{m}^{2}$ ). At sacrifice at 42 days of age, anticoagulated blood samples were taken from 30 males and 30 females from each treatment, the hematocrit was determined by the micro method and the $\mathrm{H} / \mathrm{L}$ ratio was calculated by the count of heterophile and lymphocyte by smear. Data were evaluated with the Mann-Whitney $U$ test separately for each sex and season with density as source of variation. In females, the hematocrit value showed significant differences in winter (standard $32.5 \mathrm{vs}$ reduced $31 \%$; $\mathrm{p}=0.035$ ) and marginally significant in spring (standard 31 vs reduced $30 \% ; \mathrm{p}=0.093$ ). In males, the $\mathrm{H} / \mathrm{L}$ ratio difference was marginally significant with higher value in reduced density in summer $(\mathrm{p}=0.093)$ and in females in summer $(\mathrm{p}=0.08)$ and in winter ( $\mathrm{p}=0.077$ ). It is concluded that the modification of the stocking density in winter causes environmental conditions that led to an increase in the hematocrit in chickens housed at a higher density, a response that was more evident in the case of females, while the decrease in density produces increases in $\mathrm{H} / \mathrm{L}$ ratio not compatible with situations of chronic stress.
\end{abstract}

Key words: broiler chickens, animal welfare, stress, haematology. 


\section{INTRODUCCIÓN}

Entre las normas de manejo aplicadas para responder a la creciente demanda de carne aviar en los sistemas de producción intensivos, una de las más utilizadas es la densidad de alojamiento ${ }^{1}$. Ella expresa la capacidad de carga en cantidad de aves o $\mathrm{kg}$ de aves por unidad de superficie que puede admitir un galpón y se encuentra estrechamente relacionada con el mantenimiento de las condiciones ambientales adecuadas para alcanzar determinados objetivos productivos y de bienestar.

El proceso de selección en aves productoras de carne ha dado por resultado cambios anatómicos, fisiológicos y metabólicos que aumentan su sensibilidad a las variaciones en la calidad del aire provisto por el galpón, en particular en los genotipos de rápido crecimiento ${ }^{8}$.

Esas variaciones, en parte derivadas de la asignación del espacio, influyen sobre el bienestar animal cuyo deterioro se refleja en distintos indicadores entre los que se encuentran los valores del hematocrito y la relación heterófilo-linfocito $(\mathrm{H} / \mathrm{L})^{15}$.

La presión parcial de oxígeno y la concentración de dióxido de carbono, las bajas y altas temperaturas $\mathrm{y}$ el aumento de la humedad inducen un alto consumo de oxígeno ${ }^{11}$. Estos factores del entorno avícola, sumado al aumento de los niveles de amoníaco en el ambiente, han demostrado ser causas de incrementos de la hemoglobina, de los eritrocitos y del hematocrito, respuestas mediadas por variaciones en la eritropoyesis.

Es así que ante el estado de hipoxia con grados variables de duración e intensidad, se ha señalado al hematocrito como uno de los indicadores más confiables para detectarlo ${ }^{7}$. Los valores de los índices hematológicos, como número y tamaño de los glóbulos rojos presentan, además, variaciones marcadas en relación con el sexo de las aves, lo que hace necesaria la consideración y el análisis de ambos sexos por separado ${ }^{14}$

El efecto que produce la asignación de espacio también ha sido relacionado con otras respuestas fisiológicas al estrés causado por la alta densidad de crianza ${ }^{2,3}$.

Los pollos para carne sometidos a situaciones estresantes presentan cambios comportamentales y fisiológicos, entre los que se incluyen modificaciones en el número de células de la serie blanca de la sangre, particularmente en el perfil de heterófilos y linfocitos circulantes, lo que transforma a la $\mathrm{H} / \mathrm{L}$ en un indicador confiable de estrés y pérdida de la homeostasis inmunológica inducida por los factores de tensión ${ }^{13}$.

El objetivo de este trabajo fue evaluar el efecto de la densidad de alojamiento sobre el valor del hematocrito y de la $\mathrm{H} / \mathrm{L}$ en pollos machos y hembras criados en galpones black out en las cuatro estaciones del año.

\section{MATERIAL Y MÉTODOS}

Instalaciones. El trabajo se llevó a cabo en una granja comercial del noreste de la Provincia de Santa Fe, Argentina, en dos galpones oscurecidos, de venti- lación forzada, tipo túnel. Estos tenían iguales dimensiones, $150 \mathrm{~m}$ de longitud por $14 \mathrm{~m}$ de ancho y una superficie de $2.100 \mathrm{~m}^{2}$, orientados en sentido este-oeste, con piso de tierra apisonada. El sistema de ventilación tenía ocho extractores con cono metálico de 54 pulgadas y dos extractores con cono plástico de 57 pulgadas. El sistema combinado de refrigeración evaporativa incluía dispositivos de nebulización y paneles evaporativos de $0,15 \mathrm{~m}$ de espesor, compuestos por láminas de cartón superpuestas.

Animales. Se utilizaron 54.000 pollos Cobb500 de un día de edad, que fueron alojados en lotes mixtos durante 42 días en cada crianza. El programa de alimentación incluyó cuatro dietas para todo el ciclo de producción, formuladas con materias primas convencionales (maíz y soja) y ajustadas a la demanda de las aves según su edad: iniciador medicado (0-6 días), crecimiento (724 días), terminador (25-35 días) y terminador final parrilleros (36-42 días). El alimento se presentó en forma de pellets. El programa sanitario incluyó vacunaciones contra coccidiosis y enfermedad Newcastle/bronquitis infecciosa por aspersión, y enfermedad Gumboro/ Marek por vía subcutánea, las que se realizaron en la planta de incubación al día de nacimiento, previo a su traslado a la granja.

Diseño experimental. El trabajo se realizó durante cuatro crianzas, correspondientes a cada estación del año. Se trabajó con dos densidades: estándar (14 po$1 \mathrm{los} / \mathrm{m}^{2}$ ) y reducida (12 pollos $/ \mathrm{m}^{2}$ ). En cada estación, al momento del sacrificio a los 42 días de edad, se obtuvieron alícuotas de sangre de 30 machos y 30 hembras de cada densidad en tubos de vidrio con EDTA. Para la determinación del hematocrito (Hto, método micro) se homogeneizó la muestra de sangre, se cargaron los tubos micro por capilaridad, se sellaron a fuego, y se llevaron a la microcentrífuga a $12.000 \mathrm{rpm}$ durante 2 min al cabo de los cuales se leyeron con un ábaco para microhematocrito. Para la determinación de la $\mathrm{H} / \mathrm{L}$ se realizó un frotis y se tiñó con May Gründwald-Giemsa, se observó en el microscopio óptico con un objetivo $100 \mathrm{X}$, se contaron 100 células blancas, se clasificaron y se obtuvo la relación $\mathrm{H} / \mathrm{L}$ como el cociente entre cantidad de heterófilos y linfocitos.

Análisis estadístico. El efecto densidad de alojamiento sobre el hematocrito y la H/L se evaluó mediante la prueba U de Mann-Whitney de comparación de medianas, en forma separada para cada sexo y en cada estación del año.

\section{RESULTADOS}

En la Tabla 1 se consignan los resultados del hematocrito en machos para las cuatro estaciones del año según densidad. Como se puede advertir, no se observaron diferencias significativas asociadas a la densidad de alojamiento en ninguna de las estaciones del año. 
Por otra parte (Tabla 2), el valor del hematocrito mostró diferencias significativas a favor de las hembras alojadas con densidad estándar en el invierno y una diferencia marginalmente significativa para este grupo experimental en el ensayo de primavera.

En la Tabla 3 se observan los resultados obtenidos para la relación heterófilos/linfocitos en machos, en las cuatro estaciones del año. El efecto densidad de alojamiento ${ }^{10}$ produjo una diferencia marginalmente significativa a favor de los pollos alojados con menor densidad en el verano, en tanto que en las demás estaciones no se manifestaron dichas diferencias.

La Tabla 4 expone que en las hembras se observaron diferencias marginalmente significativas a favor del grupo con densidad reducida en el verano y en el invierno.

\section{DISCUSIÓN}

El hematocrito (volumen del paquete globular expresado como porcentaje del total de la sangre), se ve modificado por cambios en el ambiente, entre los cuales la temperatura y la calidad del aire, intimamente relacionadas con la cantidad de aves por metro cuadrado, se han señalado como posibles causas de fluctuaciones ${ }^{4}$.

En el presente trabajo se hipotetizó que el alojamiento de pollos parrilleros de ambos sexos a distintas densidades en las cuatro estaciones del año produce modificaciones en los valores del hematocrito, que reflejan un esfuerzo de adaptación de las aves al ambiente.

En términos generales, independientemente del tratamiento al que fueron asignadas las aves, los valores obtenidos para el hematocrito coinciden con los reportados como normales por otros autores en condiciones estándar de crianza y con los programas de alimentación aplicados a este tipo de aves con la misma edad ${ }^{18}$

En las condiciones del presente estudio, la densidad de alojamiento no produjo diferencias significativas en los machos. Sin embargo, en las hembras, la densidad estándar se reflejó en mayores valores del hematocrito en invierno, lo que sugiere que el perfil hemático de las
Tabla 1. Hematocrito en pollos parrilleros machos según densidad de alojamiento en las cuatro estaciones del año.

\begin{tabular}{lcccc}
\hline estación & densidad estándar & densidad reducida & $\begin{array}{c}\text { estadístico Ude } \\
\text { Mann-Whitney }\end{array}$ & $\begin{array}{c}\text { probabilidad } \\
\text { asociada }\end{array}$ \\
\hline verano & $31(27,5-33,5)$ & $31(29-34)$ & 316,0 & 0,275 \\
otoño & $37(33,8-39)$ & $36,5(33,3-39)$ & 344,5 & 0,370 \\
invierno & $32(30-33)$ & $31(29,5-32,5)$ & 331,0 & 0,115 \\
primavera & $32(29-34)$ & $32,5(29-35)$ & 385,0 & 0,169 \\
\hline
\end{tabular}

Tamaño muestral: $\mathrm{n}=30$ aves por densidad.

Todos los valores corresponden a la mediana (rango intercuartílico)

Tabla 2. Hematocrito en pollos parrilleros hembras según densidad de alojamiento en las cuatro estaciones del año.

\begin{tabular}{lcccc}
\hline estación & densidad estándar & densidad reducida & $\begin{array}{c}\text { estadístico U de } \\
\text { Mann-Whitney }\end{array}$ & $\begin{array}{c}\text { probabilidad } \\
\text { asociada }\end{array}$ \\
\hline verano & $31(29-34)$ & $33(30-35)$ & 326,0 & 0,142 \\
otoño & $32(30,8-35)$ & $32(30,5-35)$ & 385,5 & 0,227 \\
invierno & $32,5(30-34)$ & $31(29-32,3)$ & 304,5 & 0,035 \\
primavera & $31(30-33)$ & $30(28,5-33)$ & 348,0 & 0,093
\end{tabular}

Tamaño muestral: $\mathrm{n}=30$ aves por densidad.

Todos los valores corresponden a la mediana (rango intercuartílico).

Tabla 3. Relación heterófilo-linfocito en pollos parrilleros machos según densidad de alojamiento en las cuatro estaciones del año.

\begin{tabular}{lcccc}
\hline estación & densidad estándar & densidad reducida & $\begin{array}{c}\text { estadístico Ude } \\
\text { Mann-Whitney }\end{array}$ & $\begin{array}{c}\text { probabilidad } \\
\text { asociada }\end{array}$ \\
\hline verano & $0,32(0,23-0,48)$ & $0,38(0,31-0,52)$ & 233,5 & 0,093 \\
otoño & $0,37(0,25-0,48)$ & $0,44(0,31-0,59)$ & 279,0 & 0,110 \\
invierno & $0,33(0,25-0,47)$ & $0,33(0,25-0,52)$ & 399,0 & 0,374 \\
primavera & $0,38(0,31-0,51)$ & $0,44(0,36-0,57)$ & 368,5 & 0,159 \\
\hline
\end{tabular}

Tamaño muestral: $\mathrm{n}=30$ aves por densidad.

Todos los valores corresponden a la mediana (rango intercuartílico).

Tabla 4. Relación heterófilo-linfocito en pollos parrilleros hembras según densidad de alojamiento en las cuatro estaciones del año.

\begin{tabular}{lcccc}
\hline estación & densidad estándar & densidad reducida & $\begin{array}{c}\text { estadístico Ude } \\
\text { Mann-Whitney }\end{array}$ & $\begin{array}{c}\text { probabilidad } \\
\text { asociada }\end{array}$ \\
\hline verano & $0,40(0,31-0,70)$ & $0,52(0,38-0,68)$ & 259,0 & 0,080 \\
otoño & $0,37(0,24-0,52)$ & $0,29(0,21-0,46)$ & 356,0 & 0,215 \\
invierno & $0,31(0,23-0,38)$ & $0,34(0,30-0,41)$ & 292,0 & 0,077 \\
primavera & $0,40(0,31-0,51)$ & $0,34(0,27-0,46)$ & 357,0 & 0,120 \\
\hline
\end{tabular}

Tamaño muestral: $\mathrm{n}=30$ aves por densidad.

Todos los valores corresponden a la mediana (rango intercuartílico).

mismas se vio afectado por diferencias en el entorno derivadas de la menor asignación de espacio como ha sido reportado por otros autores ${ }^{17}$.

La disminución de la tensión de oxígeno en el aire ha sido señalada como causa de aumento en el hematocrito en pollos, lo que pone de relieve la importancia 
del control de las condiciones ambientales como factores críticos en la producción intensiva ${ }^{16}$.

Se ha señalado que el aumento de los eritrocitos y por lo tanto del hematocrito en las hembras, es consecuencia de su reducida concentración de hemoglobina corpuscular media en los glóbulos rojos, que se ve compensado por el aumento en su cantidad, situación que se agudiza cuando la demanda de oxígeno no es satisfecha adecuadamente ${ }^{12}$

Si bien, en general, los resultados observados coincidieron con los informados como normales para este tipo de aves ${ }^{18}$, la reducción de la densidad de alojamiento produjo aumentos de la $\mathrm{H} / \mathrm{L}$ en los machos en verano y en las hembras en verano e invierno. En los pollos parrilleros la relación heterófilo/linfocito $(\mathrm{H} / \mathrm{L})$ ha sido señalada como un indicador preciso de situaciones de estrés a largo plazo con un valor basal de 0,45 y su elevación se asocia a una respuesta del sistema inmune al estrés crónico ${ }^{6}$.

La relación heterófilo/linfocito es un indicador confiable de estrés en las aves, aunque algunos estudios demuestran que es independiente de la densidad ${ }^{20}$. La baja densidad de alojamiento ha sido reportada como causa de aumento en la relación heterófilo/linfocito lo cual sugiere la complejidad de la dinámica social en las aves de corral. Esto hace necesario una correcta interpretación de los valores obtenidos para la variable $\mathrm{H} / \mathrm{L}$ y su adecuada valoración en conjunto con otros parámetros afectados por el entorno y las prácticas de manejo ${ }^{5}$.

El efecto de la densidad sobre la relación heterófilo/ linfocito como un indicador del nivel de estrés en las aves es controversial debido a que los resultados dependen de una variedad de factores relacionados con las condiciones ambientales que potencialmente pueden afectar en forma adversa el sistema inmune de los animales.

Otros investigadores evaluaron el efecto de varias densidades y de la disponibilidad de perchas sobre el estado inmune en pollos parrilleros comerciales en densidades de crianza de 10,15 y 20 aves $/ \mathrm{m}^{2}$ sin observar diferencias en la $\mathrm{H} / \mathrm{L}^{9}$. Estos autores señalaron que la falta de diferencias significativas al final del ciclo entre las distintas densidades fue la consecuencia de la alta variabilidad de este indicador al momento de la faena, la cual podría ser disminuida mediante el muestreo en distintos momentos a lo largo del ensayo.

Para contrastar esta hipótesis, otros científicos condujeron ensayos tendientes a evaluar los efectos de la densidad de alojamiento sobre la respuesta fisiológica adaptativa al aumento de la misma, sin observar cambios significativos para estas variables a lo largo del tiempo ${ }^{19}$.

Se concluye que, en las condiciones del presente trabajo, la modificación de la densidad de alojamiento en invierno produjo condiciones ambientales que derivaron en un aumento del hematocrito en los pollos alojados a mayor densidad, respuesta que fue más evidente en el caso de las hembras. Los resultados del presente estudio permiten concluir que la densidad produce modificaciones en la $\mathrm{H} / \mathrm{L}$, no compatibles con situaciones de estrés crónico.

\section{REFERENCIAS}

1. Abudabos AM et al. 2013. Impacts of stocking density on the performance and welfare of broiler chickens. Ital J Anim Sci 12: 1, 11

2. Arruda JN et al. 2016. Live performance, carcass yield, and welfare of broilers of different genetic strains reared at different housing densities. Braz J Poultry Sci 18: 1, 141152.

3. Bedanova I et al. 2007. Stress in broilers resulting from shackling. Poult Sci 86: 6, 1065-1069.

4. Biswas A. 2019. Pulmonary hypertension syndrome in broiler chickens: a review. Veterinarski Arhiv 89: 5, 723 734.

5. Cravener TL, Roush WB, Mashaly MM. 1992. Broiler production under varying population densities. Poult Sci 71: 3, 427-433.

6. Gross WB, Siegel HS. 1983. Evaluation of the heterophil/ lymphocyte ratio as a measure of stress in chickens. Avian Dis 27: 4, 972-979.

7. Hassanzadeh M, Buyse J, Toloei T, Decuypere E. 2013. Ascites syndrome in broiler chickens: A review on the aspect of endogenous and exogenous factors interactions. $J$ Poult Sci 51: 3, 229-241.

8. Havenstein, GB, Ferket, PR, Qureshi MA. 2003. Growth livability, and feed conversion of 1957 versus 2001 broilers when fed representative 1957 and 2001 broiler diets. Poult Sci 82:1500-1508

9. Heckert RA, Estevez I, Russek CE, Pettit RR. 2002 Effects of density and perch availability on the immune status of broilers. Poult $S_{c i}$ 81: 4, 451-457.

10. Lallo CH, Williams M, Campbell M, Palmer DW. 2012 The effect of stocking density on the performance and economic implications for broilers grown to 42 days in open sided house in Trinidad. Trop Agric (Trinidad) 89: 3, 170.

11. Moura AS et al. 2016. Quantitative trait loci with sexspecific effects for internal organs weights and hematocrit value in a broiler-layer cross. J Appl Genet 57: 2, 215-224.

12. Nowaczewski S, Kontecka H. 2012. Haematological indices, size of erythrocytes and haemoglobin saturation in broiler chickens kept in commercial conditions. Anim Sci Pap \& Rep 30: 2, 181-190.

13. Qaid M, Albatshan H, Shafey T, Hussein E, Abudabos AM. 2016. Effect of stocking density on the performance and immunity of 1 to $14 \mathrm{~d}$ old broiler chicks. BrazJPoultry Sci 18: 4, 683-692.

14. Rosario MF, Silva MA, Martins E, Savino VJ, Coelho AA. 2000. Influência do genótipo e do sexo sobre o valor hematócrito em galinhas reprodutoras pesadas. Braz $J$ Poultry Sci 2: 3, 281-286.

15. Rauw WM, Gomez RL. 2015. Genotype by environment interaction and breeding for robustness in livestock. Front Genet 6: 310, 1-15

16. Scheele CW, Vanderklis JD, Kwakernaak C, Buys N, Decuypere E. 2003. Haematological characteristics pre- 
dicting susceptibility for ascites. 1. High carbon dioxide tensions in juvenile chickens. Br Poult Sci 44: 3, 476-483.

17. Scheele CW, Vanderklis JD, Kwakernaak C, Buys N, Decuypere E. 2003. Haematological characteristics predicting susceptibility for ascites. 2. High haematocrit values in juvenile chickens. Br Poult Sci 44: 3, 484-489.

18. Talebi A, Asri RS, Rozeh CR, Sahraei R. 2005. Comparative studies on haematological values of broiler strains (Ross, Cobb, Arbor-acres and Arian). Int J Poult Sci 4: 8, 573-579.
19. Thaxton JP et al. 2006. Stocking density and physiological adaptive responses of broilers. Poult Sci 85: 819-824.

20. Türkyilmaz MK. 2008. The effect of stocking density on stress reaction in broiler chickens during summer. Turk $J$ Vet Anim Sci 32: 1, 31-36

\section{SIR scimaso $^{\text {sen }}$ S Journal \& Country Rank}

\section{Powered by Scopus}

\section{Revista Veterinaria mantiene su índice de impacto}

Noticias de Scimago Research Group (Scimago Journals \& Country Ranks, Scopus-Elsevier) comunican que la publicación de nuestra casa, Revista Veterinaria, aumentó su índice de impacto. El índice SJR mide la influencia científica (impacto) del artículo de una revista, expresando cuán importante es el "artículo promedio" de la publicación en la discusión científica global (sistema Thomson Reuters).

Para nuestra revista, tal indicador había sido de 0,03 entre 2008 y 2011, aumentando a 0,05 en 2012, a 0,11 en 2013, a 0,108 en 2015, a 0,100 en 2017 y a 0,114 (Research Gate) en 2018, último período evaluado Asimismo, surge para nuestra publicación un sostenido descenso del indicador que relaciona "citas versus autocitas", demostrando que los autores de los artículos están abandonando la práctica de citar sus propias publicaciones anteriores.

Por último, se advierte que según este portal, nuestra "Revista Veterinaria" continúa siendo la única publicación de esta rama de la ciencia que posee índice de impacto en Argentina. Para el resto del cono sur tal distinción recae en Brasil, Chile, Colombia y Venezuela. En revistas de veterinaria, a nivel mundial el mayor índice de impacto (2,911 puntos) recae en Annual Review of Animal Biosciences (Estados Unidos).

Fuente:

http://www.scimagojr.com/journalrank.php?area

Retrieved September, 2019. 